\title{
Dislipidemia como factor de riesgo para enfermedad cerebrovascular: estudio de casos y controles
}

Juan Lorgio Castillo Castillo, ${ }^{1, a}$, Teodoro Julio Oscanoa Espinoza ${ }^{2, b, c}$

\section{RESUMEN}

Objetivo: Determinar si la dislipidemia es un factor de riesgo independiente para enfermedad cerebrovascular.

Material y métodos: Se realizó un estudio de casos y controles pareados por edad y sexo. Los casos estuvieron conformados por pacientes con diagnóstico clínico y tomográfico de enfermedad cerebrovascular y los controles por pacientes con enfermedad distinta. A ambos grupos se les realizó dosaje sérico de colesterol total, LDLc y triglicéridos en las primeras 24 horas de su ingreso al hospital.

Resultados: El total de pacientes estudiados fueron 160 (80 casos y 80 controles). La media para los casos fue de $64,92 \pm$ 11,58 años y los controles $64,97 \pm 11,42$ años; el $63,75 \%$ fueron del género masculino y $36,25 \%$ del género femenino ( $p=$ 0,798, no significativo). La media de colesterol sérico, triglicéridos y LDLc en los casos fueron 191,4, 130,50 y 120,41 mg/dl y en los controles 210,16, 167,07 y 132,55; con $p=0,008,0,001$ y 0,060; respectivamente. El Odds ratio entre los casos y controles demuestra que la dislipidemia no incrementa el riesgo para enfermedad cerebrovascular (OR: 0,308).

Conclusión: La dislipidemia no es un factor de riesgo independiente para enfermedad cerebrovascular.

Palabras clave: enfermedad cerebrovascular; dislipidemia, colesterolemia, trigliceridemia, factores de riesgo.

\section{Dyslipidemia as risk factor for cerebrovascular disease: case-control study}

\section{ABSTRACT}

Objetive: To determine whether dyslipidemia is an independent risk factor for cerebrovascular disease.

Material and methods: A case-matched controls by age and sex was performed. The cases were composed of patients with clinical and tomographic diagnosis of cerebrovascular disease and controls for patients with different disease. Both groups underwent dosage serum total cholesterol, LDL cholesterol and triglycerides in the first 24 hours of admission to hospital.

Results: A total of 160 patients were studied ( 80 cases and 80 controls). The average for cases was $64.92 \pm 11.58$ years and $64.97 \pm 11.42$ years controls; $63.75 \%$ were of male and $36.25 \%$ females ( $p=0.798$, not significant). Mean serum cholesterol, triglycerides and LDL cholesterol in the cases were $191.4,130.50$ and $120.41 \mathrm{mg} / \mathrm{dl}$; with $p=0.008,0.001$ and 0.060 in cases and controls respectively. The odds ratio between cases and controls demonstrated that dyslipidemia does not increase the risk for cerebrovascular disease (OR: 0.308).

Conclusion: dyslipidemia is not an independent risk factor for cerebrovascular disease.

Key words: cerebrovascular disease; dyslipidemia, cholesterolemia, triglycerides, risk factors.

1. Médico neurólogo, médico asistente.

2. Doctor en Medicina.

a. Hospital de apoyo III. Sullana, Perú.

b. Departamento de Ciencias Dinámicas, Facultad de Medicina, Universidad Nacional Mayor de San Marcos.

c. Instituto de Investigación, Facultad de Medicina Humana, Universidad de San Martín de Porres. 


\section{INTRODUCCIÓN}

La enfermedad cerebro vascular (ECV) es un problema de salud pública, constituyendo la segunda causa de muerte y la tercera de discapacidad en el mundo ${ }^{(1)}$. En las últimas cuatro décadas, la incidencia se ha incrementado en los países de bajos y medianos ingresos económicos. En Perú, se ha reportado una prevalencia de $6,8 \%$ en la zona urbana y $2,7 \%$ en la zona rural ${ }^{(1,2)}$. Así mismo, el Ministerio de Salud informó un incremento en la mortalidad entre los años 2000 y $2006^{(2)}$. En las mujeres peruanas la ECV es la tercera causa de muerte $(4,6 \%$ y en los varones la cuarta causa $(3,9 \%)^{(3)}$. Castañeda-Guarderas A et al realizaron un estudio descriptivo retrospectivo en pacientes hospitalizados en el Hospital Nacional Cayetano Heredia entre los años 2000 a 2009 encontrando 2225 pacientes con diagnóstico de ECV; de los cuales 1071 presentaron isquemia y 554 hemorragia ${ }^{(2)}$.

Los factores epidemiológicos y las características clínicas de la ECV varían de acuerdo a factores ambientales, raciales, de género y socioculturales. Este hecho toma mayor relevancia debido al creciente aumento de personas con ECV en los países de bajos y medianos ingresos, siendo aún más grave el hecho de que en estas poblaciones cada vez se presentan eventos a más temprana edad y con mayor mortalidad $^{(4-7)}$.

Existe una serie de factores de riesgo cerebrovascular modificables, como la hipertensión arterial (HTA), la diabetes mellitus (DM), dislipidemias y la fibrilación auricular (FA), cuyo reconocimiento y control es fundamental para disminuir la aparición y recurrencia de $E_{C V^{(4,6)}}$. Diversos estudios han señalado la importancia de los triglicéridos (TG) en la aparición del ictus isquémico. Así, en el Copenhagen City Heart Study: “un estudio prospectivo sobre 13,956 sujetos seguidos durante más de 30 años, la incidencia acumulada de ictus isquémico se incrementó con el aumento de los niveles de TG postprandiales, de forma que los individuos con niveles de triglicéridos más elevados tenían un riesgo entre 2,5 a 3,8 veces mayor que los sujetos con valores más bajos de triglicéridos"(4). También, en un meta-análisis sobre 64 ensayos controlados aleatorizados que incluía a 195,488 pacientes, se halló una asociación estadísticamente significativa entre los niveles de TG basales y el riesgo de ictus, con un aumento del riesgo relativo ajustado de 1,05 por cada $10 \mathrm{mg} / \mathrm{dL}$ de aumento en los TG ${ }^{(5)}$. Otros estudios establecen una relación directa entre las cifras de colesterol total (CT) y el riesgo de ictus isquémico ${ }^{(7)}$.

Sin embargo; algunos estudios observacionales no han demostrado una clara asociación entre hiperlipidemias y ECV ${ }^{(8)}$. Así en el estudio de Framingham, dicha relación sólo fue evidente en un subgrupo de mujeres jóvenes. Por otro lado en el Múltiple Risk Factor Intervention Trial (MRFIT), hubo una correlación entre niveles de $\mathrm{CT}$ y riesgo de ECV isquémico pero al mismo tiempo se observó una asociación inversa para el caso del ECV hemorrágico ${ }^{(9,10)}$. Es así que en la era pre-estatinas algunos estudios sugerían que bajar los niveles lipídicos podría aumentar el riesgo de homorragia intracraneal. La falta de correlación entre lípidos y ECV también se verificaba en un metaanálisis que había incluido 45 cohortes prospectivas, seguidas por 16 años. En ese sentido, la ausencia de una fuerte relación entre colesterol plasmático e incidencia de ECV ha sido una verdadera paradoja en la epidemiología cardiovascular ${ }^{(10)}$.

El Adult Treatment Panel III Guidelines considera a los TG como un factor de riesgo independiente para $E C V^{(9,10)}$. No obstante, hay controversia en relación con esta aseveración ${ }^{(9)}$. Un estudio prospectivo publicado recientemente de casi 14.000 pacientes seguidos por 33 años en Dinamarca, correlaciona niveles altos de TG sin ayuno (entre 289 y $442 \mathrm{mg} / \mathrm{dl}$ ) con alta incidencia de ECV, especialmente en el sexo femenino; pero, no se encontró correlación con los niveles de colesterol, excepto con cifras de colesterol total superior a $348 \mathrm{mg} / \mathrm{dl}^{(10)}$.

El conocimiento actual de los factores de riesgo asociados con el desarrollo de ECV, se basa en su mayoría en datos obtenidos de estudios realizados en el extranjero ${ }^{(8)}$; pocos datos se han publicado respecto a la población peruana, especialmente en la determinación de la dislipidemia como factor de riesgo.

El objetivo del presente estudio es determinar si la dislipidemia es un factor de riesgo independiente para ECV en pacientes del Hospital de Apoyo III Sullana en el periodo de enero 2014 a diciembre del 2015.

\section{MATERIAL Y MÉTODOS}

Se realizó un estudio de tipo prospectivo, analítico, observacional, de casos y controles, apareados por edad y sexo. El grupo de estudio estuvo constituido 
por pacientes mayores de 35 años, que ingresaron a emergencia u hospitalización de los servicios de Medicina del Hospital de Apoyo III Sullana entre enero 2014 a diciembre del 2015, con el diagnóstico de ECV en las primeras 72 horas de evolución.

Casos: Pacientes con enfermedad cerebrovascular aguda, considerándose como tal el ictus isquémico o los hemorrágicos, diagnosticados por la clínica y confirmados con estudios tomográficos.

Controles: Los controles fueron seleccionados entre los pacientes que acudieron por emergencia u hospitalización de medicina con ausencia de elementos clínicos o imagenológicos de enfermedad cerebrovascular aguda. Para cada caso se seleccionó un control apareado por edad (+/- 2 años) y mismo género.

Criterios de Selección: Se definió accidente cerebrovascular basándose en los hallazgos clínicos convencionales de presentación y evolución neurológica ${ }^{(10)}$. Se incluyeron: pacientes con aceptación voluntaria verbal y escrita (del paciente o un familiar a cargo del paciente) para la participación en el estudio (Consentimiento informado). Se excluyeron: los pacientes con diagnóstico de ictus previo, consumo de hipolipemiantes previo al ingreso hospitalario y negativa verbal a participar en el estudio.

Para definir a los pacientes dislipidémicos se aplicaron los actuales criterios de diagnóstico: cifras colesterol total $\geq 200 \mathrm{mg} / \mathrm{dL}$, triglicéridos $\geq 150$ $\mathrm{mg} / \mathrm{dL}$, LDLc $\geq 100 \mathrm{mg} / \mathrm{dL}^{(4,6,7,9)}$. Se considera dislipidemia si el paciente presenta uno o más de estos valores; en muestra tomada en las primeras 24 horas de ingreso y en ayunas entre las 7 a 8 am.

La muestra fue seleccionada de forma aleatoria, $1 \mathrm{de}$ cada 3 pacientes que reunieron los criterios para el estudio.

Análisis Estadístico: Los datos fueron analizados en el programa SPSS para Windows versión 17.0. Los resultados de las variables cualitativas se expresan en frecuencias absolutas y porcentuales, y los resultados de las variables cuantitativas en forma de promedios y desviación estándar. Se aplicó la prueba Chi cuadrado para determinar asociación o independencia del factor de riesgo en relación con los grupos de estudio, considerándose significativo si $\mathrm{p}<$ 0,05 . Se calculó el Odds ratio $(I C=95 \%)$ para determinar el riesgo entre la dislipidemia y los grupos de estudio.

\section{RESULTADOS}

El total de pacientes estudiados fueron 160 (80 casos y 80 controles). De los casos, 51 (63,75\%) fueron del género masculino y $29(36,25 \%)$ del género femenino $(p=0,798)$. La edad promedio para los casos fue de $64,92 \pm 11,58$ y para los controles fue $64,97 \pm 11,42$ años. La mayoría de los pacientes con ECV corresponden al grupo entre los 55 a 65 años (Tabla 1).

Tabla 1. Distribución de casos y controles según edad. Hospital de Apoyo III Sullana

\begin{tabular}{|c|c|c|c|c|c|c|}
\hline \multicolumn{7}{|c|}{ GRUPO } \\
\hline \multirow[t]{2}{*}{ Edad (años) } & \multicolumn{2}{|c|}{ Casos } & \multicolumn{2}{|c|}{ Controles } & \multicolumn{2}{|c|}{ Total } \\
\hline & $\mathrm{n}$ & $\%$ & $n$ & $\%$ & $n$ & $\%$ \\
\hline $35-45$ & 03 & 3,75 & 02 & 2,5 & 05 & 3,125 \\
\hline $45-55$ & 12 & 15,00 & 13 & 16,25 & 25 & 15,625 \\
\hline $55-65$ & 26 & 32,50 & 23 & 28,75 & 49 & 30,625 \\
\hline $65-75$ & 21 & 26,25 & 23 & 28,75 & 44 & 27,50 \\
\hline $75-85$ & 15 & 18,75 & 16 & 20,00 & 31 & 19,375 \\
\hline$\geq 85$ & 03 & 3,75 & 03 & 3,75 & 06 & 3,75 \\
\hline TOTAL & 80 & 100 & 80 & 100 & 160 & 100 \\
\hline
\end{tabular}


La isquemia cerebral se presentó en 59 (73,75\%) de los casos y la hemorragia intracerebral en $21(26,25 \%)$ de los casos.

\section{Dislipidemia}

La dislipidemia estuvo presente en 61 (76,25\%) de los pacientes con ECV y fue más frecuente en el grupo menor de 65 años de edad $(38,75 \%)$.

La dislipidemia fue más frecuente en la isquemia cerebral y en el género masculino; pero sin diferencias estadísticamente significativas (Tabla 2).

Tabla 2. Distribución de pacientes según género, tipo de ECV y dislipidemia

\begin{tabular}{|llccccc|}
\hline \multirow{2}{*}{ Variable } & & \multicolumn{2}{c}{ Dislipidemia } & Total & P valor \\
\cline { 3 - 5 } GÉNERO & Presente & Ausente & & \\
& Femenino & 48 & 10 & 58 & 0.798 \\
\hline TIPO ECV & Masculino & 86 & 16 & 102 & \\
\hline & Isquemia & 45 & 14 & 59 & 0.994 \\
\hline
\end{tabular}

La diferencia de promedios en el colesterol total, triglicéridos y LDLc fue mayor en el grupo control, con diferencias estadísticamente significativas en colesterol total y triglicéridos (Tabla 3).

Tabla 3. Promedios y desviación estándar de los factores de riesgo en casos y controles

\begin{tabular}{|l|l|l|l|c|c|}
\hline $\begin{array}{l}\text { Factor } \\
\text { Riesgo }\end{array}$ & \multicolumn{1}{|c|}{ Grupo } & $\mathrm{n}$ & Media & $\begin{array}{c}\text { Desviación } \\
\text { típ. }\end{array}$ & $\begin{array}{c}\text { T Student } \\
\text { (IC 95\%) }\end{array}$ \\
\hline COLTOT & Caso & 80 & 191,40 & 46,61 & $\begin{array}{c}0,008 \\
(-32,65-4,87)\end{array}$ \\
\hline & Control & 80 & 210,16 & 42,24 & 0,001 \\
\hline TRIGLIC & Caso & 80 & 130,05 & 52,35 & $(-54,87-19,17)$ \\
\hline & Control & 80 & 167,07 & 61,59 & 0,060 \\
\hline LDLC & Caso & 80 & 120,41 & 40,64 & $(-24,77-0,50)$ \\
\hline & Control & 80 & 132,55 & 40,32 & \\
\hline
\end{tabular}

La dislipidemia, la hipercoleterolemia, la hipertrigliceridemia y el LDLc elevado presentan diferencias significativas entre ambos grupos; pero no incrementan el OR (Tabla 4).

Tabla 4. Análisis de los factores de riesgo para Enfermedad Cerebro-vascular

\begin{tabular}{|l|l|c|c|c|c|}
\hline \multicolumn{1}{|c|}{ Factor } & Casos & Controles & P valor & OR (IC 95\%) \\
\hline DISLIPID EM & Presente & 61 & 73 & 0,010 & $0,308(0,121-0,781)$ \\
\hline & Ausente & 19 & 07 & & \\
\hline HIPERCOLEST & Presente & 31 & 49 & 0,004 & $0,400(0,212-0,756)$ \\
\hline & Ausente & 49 & 31 & & \\
\hline HIPERTRIGL & Presente & 22 & 35 & 0,001 & $0,295(0,152-0,571)$ \\
\hline & Ausente & 58 & 45 & & \\
\hline LDLC- elevado & Presente & 57 & 68 & 0,035 & $0,437(0,200-0,956)$ \\
\hline & Ausente & 23 & 12 & & \\
\hline & & & & & \\
\hline
\end{tabular}




\section{DISCUSIÓN}

En las últimas cuatro décadas, la incidencia de la ECV ha disminuido en los países desarrollados, mientras que en Latinoamérica está aumentando ${ }^{(11)}$; por este motivo, recientemente, ha sido declarada como "enfermedad catastrófica en Iberoamérica". La Organización Mundial de la Salud (OMS), la World Federation of Neurology y la International Stroke Society han lanzado una propuesta internacional denominada "Iniciativa global de la ECV", para que, de una manera sistemática, se realicen estudios epidemiológicos, estandarizados, y se obtenga información válida para tomar posteriormente decisiones de salud pública que permitan prevenir la ECV.

Los resultados muestran una edad promedio para los casos de 64,92 \pm 11 , 58 años; similar a lo reportado por Castañeda-Guarderas $\mathrm{A}$ et $\mathrm{al}^{(2)}$ en el estudio realizado en el Hospital Nacional Cayetano Heredia entre los años 2000 a 2009 encontrando edad promedio de $64,1 \pm 16,9$ años. En Chile, Prat $H^{(10)}$ reporta edad media de 66,5 años y en Colombia, Machado-Alva J ${ }^{(12)}$ edad promedio de 64,9 $\pm 10,9$ años. En España se reporta una edad promedio de $71,8 \pm 12^{(4)}$ y $70,36 \pm 13,27$ años $^{(6)}$. Es decir, la población con ECV en el Perú y Latinoamérica es más joven que en la población europea; lo cual estaría relacionado con las variaciones regionales, raza, estilos de vida poco saludables y adicciones.

En México ${ }^{(11)}$, la ECV ha pasado de ser la cuarta causa de mortalidad general en el año 2000, a ser la tercera causa en el año 2008 y se presenta con mayor frecuencia en las mujeres $(52,2 \%$ ) que en los varones $(47,8 \%)$; similar a la reportado por MachadoAlva ${ }^{(12)}$, encontrando predominio femenino $(56,4 \%)$. En Chile ${ }^{(10)}$ y Colombia ${ }^{(8)}$ se reporta mayor frecuencia en los varones (56\% y $50,45 \%$, respectivamente). En nuestro estudio el $(63,75 \%)$ corresponden al género masculino; similar a lo reportado en otro estudio peruano ${ }^{(2)}$; en España se reporta $53,6 \%{ }^{(4)}$ y $65 \%{ }^{(13)}$. En base a este resultado nuestra población se comportaría de acuerdo con lo descrito por la AHA, la cual afirma que la incidencia de ACV es mayor en varones; sin embargo, difiere de estudio mexicano esto probablemente se deba a una mayor atención al hombre o a la mujer en el hospital donde se realizó el estudio, o a que se trate de etiologías distintas; como la del estudio brasileño que encontró ECV más frecuente en mujeres debido a microangiopatía ${ }^{(8)}$.

La isquemia cerebral es el tipo más frecuente de EVC en la mayoría de los reportes, variando entre 60 al
$86 \%^{(1,3,6,13)}$. En el presente estudio el $73,75 \%$ de los casos presentaron isquemia cerebral y el $26,25 \%$ hemorragia intracerebral; concordando con los resultados de otros investigadores nacionales ${ }^{(2)} \mathrm{e}$ internacionales ${ }^{(6,13)}$.

El papel de la dislipidemia como factor de riesgo independiente para la ECV se ha reportado en varios estudios; sin embargo, en la mayoría de ellos el principal objetivo no era la dislipidemia en sí misma, sino que esta es analizada en el marco de otros objetivos más amplios, lo cual dificulta la comparación entre ellos y la extracción de conclusiones definitivas. Su importancia radica en los efectos sobre la aceleración de la arteriosclerosis, que se potencian de forma exponencial cuando coinciden con otros factores de riesgo ${ }^{(14)}$.

El estudio realizado en Colombia( ${ }^{(8)}$ reporta dislipidemia en el $55,91 \%$ de los pacientes con ECV; similar a hispanos residentes en Estados Unidos $(58,3 \%)$ y Brasil $(57,8 \%)$. En España ${ }^{(4)}$, se reporta la presencia de dislipidemia en el $17 \%$ y mayor frecuencia en el género masculino $(p=0,048)$. En México ${ }^{(9)}$, se reporta dislipidemia de $69,7 \%$ en los hombres y $48,8 \%$ en las mujeres. En nuestro estudio la dislipidemia estuvo presente en $76,25 \%$ de los pacientes con ECV, más frecuente en el grupo entre 35-65 años de edad y en el género masculino. Casteñeda-Guarderas et al ${ }^{(2)}$ en Perú, reporta dislipidemia en el 1,8\% de la población general; pero no evalúa su presencia como factor de riesgo. Observamos, porcentaje de dislipidemia cercana a los países latinoamericanos y distintos a España. También observamos mayor frecuencia en adultos jóvenes; que probablemente esté en relación con los hábitos dietéticos, consumo de grasas saturadas, el sedentarismo y otros factores de riesgo mayores como el tabaquismo, la diabetes y la hipertensión arterial ${ }^{(9)}$.

Los resultados del presente trabajo sugieren que la dislipidemia no es un factor de riesgo independiente para ECV con OR: 0,308 (Tabla 4). Diversos estudios plantean la probabilidad de incremento del riesgo para ECV en los pacientes con dislipidemia ${ }^{(4,6)}$. Sin embargo; algunos estudios observacionales no han demostrado una clara asociación entre dislipidemias y ECV y en el estudio de Framingham, dicha relación sólo fue evidente en un subgrupo de mujeres jóvenes ${ }^{(8)}$. La falta de correlación entre lípidos y ECV también se verificaba en un metaanálisis que había incluido 45 cohortes prospectivas, seguidas por 16 años $^{(10)}$. Machado-Alva $\mathrm{J}^{(12)}$, plantea que las diferencias encontradas entre los tipos de 
dislipidemia y las ciudades colombianas, pueden ser debidas a distintos hábitos de vida y dieta entre habitantes de las regiones de un país; para lo cual, se requiere de un estudio capaz de establecer si los riesgos varían y cuáles son las reales causas de tal fenómeno. Consideramos que se debe ampliar el análisis a cada uno de los subgrupos de dislipidemia y tipos de ECV para evaluar su relevancia.

En el presente estudio el promedio de CT para los casos fue de 191,4 mg/dl y de 210,16 mg/dl para los controles; con diferencia de promedios estadísticamente significativos a favor del grupo control $(p=0,004)$, pero sin incrementar el riesgo $(\mathrm{OR}=0,400)$. Palacios $E^{(7)}$, en España, encuentra CT de $236,81 \pm 36,96 \mathrm{mg} / \mathrm{dl}$; observándose, valores más elevados a nuestros resultados. Sorganvi $\mathrm{V}$ et al ${ }^{(15)}$, en la India, reporta que los niveles de colesterol superiores a $220 \mathrm{mg} / \mathrm{dl}$ incrementan en 4 veces $(\mathrm{OR}=$ 4,115 ) el riesgo de presentar ECV isquémica; pero, no describe si la muestra fue tomada en ayunas o en postprandial. En el estudio realizado en Venezuela ${ }^{(14)}$, se encontró que la hipercolesterolemia elevó a casi tres veces (OR: 2,89) el riesgo de presentar ECV aguda. Sin embargo, la correlación directa entre niveles de colesterol total y ECV isquémico (trombótico) solo se ha podido demostrar con cifras superiores a $300 \mathrm{mg} / \mathrm{dl}$ lo que no es habitual de encontrar ${ }^{(10)}$. En el Múltiple Risk Factor Intervention Trial (MRFIT), hubo una correlación entre niveles de $\mathrm{CT}$ y riesgo de ECV isquémico; pero al mismo tiempo se observó una asociación inversa para el caso del ECV hemorrágico ${ }^{(9,10)}$. En el estudio realizado en Colombia ${ }^{(12)}$, se reporta que el riesgo de presentar hipercolesterolemia se asocia a las variables: vivir solo $(p=0,02)$, ser mujer mayor de 65 años $(p=0,02)$, poseer un riesgo de Framingham moderado o alto $(p<0,01)$ y vivir en las ciudades de Cartagena e Ibagué $(p=0,04)$. La ausencia de una fuerte correlación entre colesterol plasmático e incidencia de ECV ha planteado una controversia en la epidemiología cardiovascular ${ }^{(10)}$. Muchas explicaciones se han propuesto, por ejemplo la propia heterogenicidad de la enfermedad cerebrovascular, diferencias poblacionales en los niveles lipídicos, toma de muestra en ayunas o post prandial, seguimientos relativamente cortos y la falta de un mayor número de estudios ${ }^{(9,10)}$.

Los TG han sido implicados en la génesis de la ECV y el Adult Treatment Panel III Guidelines lo consideran como un factor de riesgo independiente ${ }^{(9,10)}$. El promedio de TG en nuestro estudio fue de 130,05 $\mathrm{mg} / \mathrm{dl}$ para los casos y de $167,07 \mathrm{mg} / \mathrm{dl}$ para los controles; con diferencia de promedios estadísticamente significativos a favor del grupo control $(p=0,001)$, pero sin incrementar el riesgo $(O R=0,295)$; similares a lo encontrado por Palacios ${ }^{(7)}$, en España, quien reporta un promedio de $139,5 \mathrm{mg} / \mathrm{dl}$. En una revisión sistemática de Leonards se revisaron 25 estudios, 13 de cohorte y 12 de caso- control, algunos correlacionaron la ECV con niveles de TG en ayunas y otros con TG sin ayuno, concluyendo que los datos son inconsistentes y se requiere estandarizar las técnicas de medición ${ }^{(10)}$. El estudio prospectivo realizado en Dinamarca, correlaciona niveles altos de TG sin ayuno (entre 289 y $442 \mathrm{mg} / \mathrm{dl}$ ) con alta incidencia de ECV, especialmente en el sexo femenino. Observamos, que estos valores son superiores a los nuestros; pero, la diferencia se puede deberse a que sus muestras han sido tomadas en pacientes sin ayuno. Machado-Alva $\mathrm{J}^{(12)}$, encuentra que el riesgo de presentar hipertrigliceridemia era superior en hombres mayores de 55 años, con diabetes mellitus, antecedentes de IAM, sobrepeso u obesidad, lo cual muestra la asociación de este trastorno y las alteraciones en el metabolismo de la glucosa en especial en hombres.

En el presente estudio el promedio de LDLc para los casos fue de $120,41 \mathrm{mg} / \mathrm{dl}$ y de $132,55 \mathrm{mg} / \mathrm{dl}$ para los controles; con diferencia de promedios estadísticamente significativos a favor del grupo control $(p=0,035)$, pero sin incrementar el riesgo $(\mathrm{OR}=0,437)$. Palacios $\mathrm{E}^{(7)}$, en España, encuentra LDLc promedio de $161,17 \pm 30,91 \mathrm{mg} / \mathrm{dl}$; es decir, un promedio superior al nuestro, lo cual podría estar en relación a diferencias en los hábitos, edad de los pacientes y periodo de ayuno.

Llama la atención el promedio de colesterol total, TG y LDLc más elevado en los controles que en los casos; para lo cual debería tenerse en cuenta la presencia de estrés físico (incluidas enfermedades intercurrentes agudas), cirugía o pérdida de peso ${ }^{(9)}$ que no fueron incluidas en las variables de nuestro estudio. Otras variables a considerar son: los resultados reportados en algunos estudios son obtenidos a partir de la revisión de historias clínicas y es posible que los datos no estuviesen registrados en estas o que la calidad de la información consignada no siempre sea completa y óptima; de la misma manera, como los datos se obtuvieron de diferentes ciudades, los resultados de mediciones clínicas y de laboratorio no estuvieron estandarizadas ${ }^{(12)}$. 
En cuanto a las limitaciones de nuestro estudio, en primer lugar, los datos se restringieron a un estudio transversal e intrahospitalario. En segundo lugar, el pequeño tamaño de la muestra; que a pesar de ser estadísticamente significativo, puede llevar a errores asociados. En tercer lugar, los criterios que hemos utilizado para el diagnóstico de dislipidemia son más exigentes que los de otros estudios, por lo que es posible que algunos pacientes dislipidémicos no hayan sido considerados como tales en otros estudios, lo que explicaría nuestra mayor frecuencia en los de casos y controles.

En conclusión, el presente estudio no encontró una asociación independiente entre la dislipidemia y la ECV; por lo que hace necesario más estudios con respecto a este tema.

\section{REFERENCIAS BIBLIOGRÁFICAS}

1. Davalos L, Málaga G. El accidente cerebrovascular en el Perú: una enfermedad prevalente olvidada y desatendida. Rev Perú Med Exp Salud Pública. 2014; 31(2): 393-401.

2. Castañeda-Guarderas A, Beltrán-Ale G, Casma-Bustamante R, Ruiz-Grosso P, Málaga G. Registro de pacientes con accidente cerebro vascular en un hospital del Perú, 2000-2009. Rev Perú Med Exp Salud Pública. 2011; 28(4):623-27.

3. Alcalde-Rabanal JE, Lazo-González O, Nigenda G. Sistema de salud de Perú. Salud Pública de México. 2011; 53(S2): S243S254.

4. Castilla-Guerra L, Fernández-Moreno C, Navas-alcántara S, Jiménez-Hernández D. Dislipemia aterogénica y riesgo residual en pacientes con ictus isquémico. Rev Neurol. 2015; 60 (9): 429-430.

5. Labreuche J, Deplanque D, Touboul PJ, Bruckert E, Amarenco P. Association between change in plasma triglyceride levels and risk of stroke and carotid atherosclerosis: systematic review and meta-regression analysis. Atherosclerosis. 2010; 212: 915.

6. Rojas D. Morbilidad y mortalidad del adulto mayor en un servicio de medicina de un hospital general del Perú. Rev Peruana de Epidemiología. 2010; 14(2): 1-17.
7. Palacio E, Viadero-Cervera R, Revilla M, Larrosa-Campo D, Acha-Salazar O, Novo-Robledo F, et al. Utilidad del tratamiento con atorvastatina $40 \mathrm{mg}$ más ezetimiba $10 \mathrm{mg}$ frente a atorvastatina $80 \mathrm{mg}$ en la reducción de los niveles de colesterol $\mathrm{LDL}$ en pacientes con ictus isquémico o ataque isquémico transitorio. Rev Neurol. 2016; 62 (5): 203-210.

8. Bonilla PH, Oliveros H, Proaños J, Espinel B, Álvarez J, Duran C, et al. Estudio de frecuencia de los factores de riesgo asociados al desarrollo de enfermedad cerebrovascular isquémica no embólica en un hospital de tercer nivel. Acta Neurol Colomb. 2014; 30(3):149-155.

9. Canalizo-Miranda E y Favela-Pérez E. Guía de práctica clínica, Diagnóstico y tratamiento de las dislipidemias. Rev Med Inst Mex Seguro Soc. 2013; 51(6):700-709.

10. Prat H. Enfermedad cerebrovascular y estatinas. Rev Chil Cardiol. 2012; 31(2): 148-151.

11. Cantu B, Ruiz SJ, Murillo BL, Chiquete E, Leon JC, Arauz A, et al. Manejo agudo y pronóstico a un año en pacientes mexicanos con un primer infarto cerebral: resultados del estudio multicéntrico PREMIER. Rev Neurol. 2010; 51 (11): 641-649.

12. Machado-Alva J, Machado-Duque M. Prevalencia de factores de riesgo cardiovascular en pacientes con dislipidemia afiliados al sistema de salud en colombia. Rev Perú Med Exp Salud Pública. 2013; 30(2):205-211.

13. Salvadó-Figueras M, Quintana M, Ribó M, Álvarez-Sabín J. Perfil de las urgencias neurovasculares en un hospital terciario: evolución entre los años 2001-2008. Rev Neurol. 2011; 53 (6): 321-328.

14. Frómeta A, Álvarez A, Sánchez S, Fonseca J, Quesada A. Factores de riesgos de la enfermedad cerebrovascular aguda. Revista Habanera de Ciencias Médicas. 2010; 9(4): 534-544.

15. Sorganvi V, Kulkarni M, Udgiri R, Kadeli D, Atharga S. Risk factors for ischemic stroke- a case control study. I.J.A.B.R. 2014; 4(1): 9-12.

Fuentes de financiamiento:

Los costos fueron cubiertos en su mayoría por el Sistema Integral de Salud (SIS), por corresponder a atenciones en Hospital del Ministerio de Salud.

Conflictos de interés:

Los autores declaran no tener ningún conflicto de interés.

\section{Correspondencia:}

Juan Lorgio Castillo Castillo

Dirección: Av. Faustino Sánchez Carrión № 1317 - El Porvenir - La Libertad.

Teléfono: 952099941

Correo electrónico: castillocastilloj@hotmail.com
Recibido: 23 de julio de 2016 Aprobado: 26 de agosto de 2016 